HEP/123-qed

\title{
Solar neutrinos, helicity effects and new affine gravity with torsion
}

\author{
Diego Julio Cirilo-Lombardo \\ Bogoliubov Laboratory of Theoretical Physics, \\ Joint Institute for Nuclear Research 141980, \\ Dubna(Moscow Region), Russian Federation*
}

(Date textdate; Received textdate; Revised textdate; Accepted textdate; Published textdate)

\begin{abstract}
New $f(R, T)$ model of gravitation, introduced previously by the author, is considered. It is based on an affine geometrical construction in which the torsion is a dynamical field, the coupling is minimal and the theory is Lorentz invariant by construction. It was shown that the Dirac equation emerges from the same space time and acquires a modification (coupling-like) of the form $\gamma^{\alpha} j \frac{1-d}{d} \gamma_{5} h_{\alpha}$, with $h_{\alpha}$ the torsion axial vector, $j$ a parameter of pure geometrical nature and $d$, the spacetime dimension. In the present work it is shown that this interaction produces a mechanism of spin (helicity) flipping, with its consequent weak symmetry violation. The cross section of this process is explicitly calculated and a logaritmical energy dependence (even at high energies) is found. This behavior is reminiscent of similar computations made by Hans Bethe in the context of neutrino astrophysics. These results are applied to the solar neutrino case and compared with similar results coming from a gravitational model with torsion of string theory type and within the standard model context respectively.
\end{abstract}

*Electronic address: diego777jcl@gmail.com, diego@theor.jinr.ru; Tel: +74 962166938/Fax: +74962165084 


\section{Contents}

I. Introduction

II. Statement of the problem

105

III. Cross-section

108

IV. Solar neutrino situation and spin-flip

109

V. Discussion and examples

111

A. Comparison with string-gravity model with torsion potential

111

B. Cross sections energy-dependent and logarithmic terms

VI. Interaction structure, axions and Nambu-Goldstone bosons

VII. Neutrino oscillation and IceCube data: constrained the torsion effect at higher energies

\section{Concluding remarks}

IX. Acknowledgements

\section{References}

\section{References}

\section{INTRODUCTION}

As is commonly suggested, solutions based on neutrino "spin flip" in the Sun's magnetic fields are proposed to explain the observed solar neutrino deficit. Dependence of the survival probability on energy and significant regeneration effect (day/night asymmetry) are not observed in solar neutrino detectors. In the last ten years, an increasing interest in the neutrino physics is shown by the scientific community. Four (probably related) aspects involving neutrinos are constantly the target of investigations: the solar neutrino problem , the CP and CPT violations effects and the anomalous momentum [3]. From the astrophysical point of view neutrino has a non-zero magnetic moment, the neutrino helicity can be 
flipped when it passes through a region with magnetic field perpendicular to the direction of propagation. It means that the left-handed neutrino that is active in SM would change into a right-handed one

In this paper, we attack mostly the first one, proposing an explanation for the solar neutrino problem, alternative to the proposals based on more standard approaches given, for example, in [16] and to the string theoretical arguments, involving a torsion potential, given for example in [18]. This motivation is justified by the very important analysis of the problem given by reference [13] where the bounds of the axial parameters are actualized and new experiments in this direction are suggested; and reference [14](by the same group) where bounds to the non-standard interactions of the muonic neutrinos and quarks are given.

The arguments on behalf of our explanation arise in a new model of gravitation based on a pure affine geometrical construction. The geometrical Lagrangian of the theory contains dynamically as main geometrical object, a generalized curvature $\mathcal{R}=\operatorname{det}\left(\mathcal{R}_{\mu}^{a}\right)(\operatorname{coming}$ from a higher dimensional group manifold generally based in $\mathrm{SU}(2,2 \mathrm{~N})$ ) and is defined as follows

$$
L_{g}=\sqrt{\operatorname{det}^{a}{ }_{\mu} \mathcal{R}_{a \nu}}=\sqrt{\operatorname{det} G_{\mu \nu}}
$$

where after the breaking of the symmetry and due to the reductivity of the geometry, the generalized curvature becomes to

$$
\mathcal{R}_{\mu}^{a}=\lambda\left(e_{\mu}^{a}+f_{\mu}^{a}\right)+R_{\mu}^{a} \quad\left(M_{\mu}^{a} \equiv e^{a \nu} M_{\nu \mu}\right)
$$

and the Lagrangian $L_{g}$ coming to

$$
\sqrt{\operatorname{Det} \mathcal{R}_{\mu}^{a} \mathcal{R}_{a \nu}}=\sqrt{\operatorname{Det}\left[\lambda^{2}\left(g_{\mu \nu}+f_{\mu}^{a} f_{a \nu}\right)+2 \lambda R_{(\mu \nu)}+2 \lambda f_{\mu}^{a} R_{[a \nu]}+R_{\mu}^{a} R_{a \nu}\right]},
$$

where $R_{\mu \nu}=R_{(\mu \nu)}+R_{[\mu \nu]}$ and $f_{\mu}^{a}$, in a sharp contrast with the tetrad field $e_{\mu}^{a}$, carries the symmetry $e_{a \mu} f_{\nu}^{a}=f_{\mu \nu}=-f_{\nu \mu}$.- see $[5,6,7]$ for more mathematical and geometrical details of the theory. As we have been previously pointed out, the original definition given by us of the Lagrangian as a measure containing $\mathcal{R}_{\mu}^{a}$ is reminiscent to have the same essence as in the pioneering unified field theories, in particular the Eddington one.

As was shown in $[5,6,7]$, in this specific model of $f(R, T)$ gravity the Dirac equation is derived from the same spacetime manifold, and acquires a modification (coupling-like) of the form

$$
\gamma^{\alpha} j \frac{1-d}{d} \gamma_{5} h_{\alpha}
$$


with $h_{\alpha}$ the torsion axial vector, $j$ a parameter of pure geometrical nature and $d$, the spacetime dimension. Here the torsion is a dynamical field, and the theory is Lorentz invariant by construction.

In the following, we will show that this interaction produces a mechanism of spin flipping and we will calculate explicitly the cross section for this process. This cross section, in sharp contrast with the string and standard model cases[18,16], depends logarithmically on the energy, even at high energies, which is a reminiscent of similar computations made by Hans Bethe time ago [2] considering astrophysical neutrinos.

The behavior of the cross section energy dependence is very important when considering solar neutrinos detected experimentally because the number of events naturally depends on the energy threshold. For example, the $\mathrm{Cl}$ detector of the Davis group has a threshold of 0.8 $\mathrm{MeV}$, with a lower count (28\%), than the KAMIOKA neutrino detector (with a threshold of $7.5 \mathrm{MeV}$ ), and the SAGE and GALLEX (with threshold of $0.23 \mathrm{MeV}$ ) have higher counts (51-62\%) $[12,19,17,1]$. The importance of this research is more that evident due the well known following reasons. With the increasing level of precision of the neutrino experiments, in high energy and in astrophysics the determination of neutrino parameters is the crucial point. Today, the main focus from the phenomenological point of view, is devoted to the determination with high precision of the oscillation parameters (as the testing of nonoscillation effects and possible subleading oscillations), non standard neutrino interactions (NSI) and as spin-flavour conversions. Although magnetic field effects, that are the first candidates to produce density fluctuations into the radiative zone of the sun, doesn't not modifies the robustness of the quantitative analysis of neutrino oscillations.

In our manuscript we focus on the case of neutrinos endowed with non-standard interactions making evident due the presence of the torsion as dynamical field. These are also a natural outcome of many neutrino mass models and can be in general of two types: flavour changing (FC) and non-universal (NU). Models of Seesaw type are the best examples models leading structures of the lepton mixing matrix that are non trivial ones. These matrix characterize the charged and neutral current weak interactions. Then, the NSI that are induced by gauge transformations can, even with massless neutrinos, CP and leptonic flavor. Models where the masses of the neutrinos coming from radiative corrections as in superunified models the NSI surely appears.

The organization of this paper is as follows: in Section II we obtain, by geometrical 
methods, the solution of a particle at rest in Minkowski spacetime (where the curvature effects are not important but torsion certainly exists). Considerations about the form of the geometrical Lagrangian of the theory and the nature (electric or magnetic) of the axial vector torsion are given with some technical details. In Section III, also making some exhaustive use of geometrical manipulations, we explicitly compute the cross section corresponding to a neutrino-hadron interaction in the presence of torsion. Section IV is devoted to the application of the formulation of the previous section to the computation of probability of spin flipping for the solar neutrinos, and comparing these results with similar results coming from a gravitational model with torsion of string theory type, and with totally antisymmetric torsion. Finally, Sections [V] and VIII are devoted to summarize the obtained results.

\section{STATEMENT OF THE PROBLEM}

Consider the approximation in which, avoiding strong curvature effects, we deal only with torsion fields. Seeking for spherical symmetric solutions, the line element under consideration will be, for instance,

$$
d s^{2}=-d t^{2}+d r^{2}+r^{2}\left(d \theta^{2}+\sin ^{2} \theta d \varphi^{2}\right)
$$

Therefore, the tensor $G_{\mu \nu}=-2 \lambda\left(g_{\mu \nu}+f_{\mu}^{a} f_{a \nu}\right)$ of the geometrical Lagrangian (1) stated in $[5,6,7]-$ reduces to $\left.G_{\mu \nu}\right|_{\text {Mink. }}=-2 \lambda\left(\eta_{\mu \nu}+f_{\mu}^{a} f_{a \nu}\right)$. To solve the equation of our problem we use the Cartan's structure method with the natural orthonormal system: $\omega^{0}=d t, \quad \omega^{1}=d r, \quad \omega^{2}=r d \theta, \omega^{3}=r \sin \theta d \varphi$, as usual for spherical symmetry. We use the Palatini principle that simultaneously determines the connection required for the spacetime symmetry and the dynamical field equations. The specific form of the action $\mathrm{S}$ (or the Lagrangian as we have been commented in the Introduction) is arbitrary but from this action $S$ necessarily we must reach the G-invariant conditions, namely, the intersection of the 4 dimensional Lorentz group $L_{4}$, the symplectic $S p(4)$ and the almost complex group $K(4))$ without prior assumption. Then, the Einstein, Dirac and Maxwell equations need to arise from $S$ as a causally connected closed system. Is very important to regard here that the antisymmetric $f$ field forming part of the geometrical construction can be associated to the physical electromagnetic field, namely $\varphi_{\mu \nu}$ by means the following relation

$$
f_{\mu \nu} \equiv \frac{1}{2} \varepsilon_{\mu \nu \rho \sigma} \bar{\varphi}^{\rho \sigma}=* \bar{\varphi}_{\mu \nu}
$$


where $\bar{\varphi}^{\mu \nu}$ is the inverse tensor to $\bar{\varphi}_{\mu \nu}$ and $*$ is the Hodge operator defined into the four dimensional spacetime. It is important to note that $f_{\mu \nu}$ is adimensional, corresponding to our choice for $g_{\mu \nu}$ in the Lagrangian of the theory. That means that, physically, there exists an "absolute field", namely $j$, that fulfills (analog to the $b$ field in the Born-Infeld theory ) the double role of homogenizing the units and put a maximum limit to the magnitude of the fields: $\frac{\varphi_{\mu \nu}}{j} \equiv \bar{\varphi}_{\mu \nu}$, this is the meaning of the bar over the physical fields. This field $j$ should play a fundamental physical role in the phenomenology of the spin flipping, as we will see when computing the cross section of the neutrino-hadron interaction. We will work, for simplicity, with $f_{\mu \nu}$ reminding that the physical electromagnetic fields $\varphi_{\mu \nu}$ are the respective dual due (6): for electric $f_{\mu \nu}$ corresponds physical magnetic $\varphi_{\mu \nu}$ and viceversa.

The axial vector $h_{\mu}$, dual of the total antisymmetric torsion field $T_{\mu \nu \varrho}$, plays a fundamental role in the Dirac equation derived in $[5,6,7]$ from the same spacetime manifold $M$. It modifies the anomalous momentum $g$ of the elementary fermionic particles and, due to the symmetries of the problem, we assume $h=h(r, \theta)$ (geometrical notation). The dynamical equations of the $f$ field are comparable in form to the Born-Infeld case $[9,10]$. Then, as a natural ansatz, $f$ will be assumed to have the form

$$
f=f_{31} \omega^{3} \wedge \omega^{1}+f_{23} \omega^{2} \wedge \omega^{3}+f_{10} \omega^{1} \wedge \omega^{0}+f_{20} \omega^{2} \wedge \omega^{0}
$$

and $\mathbb{F} \equiv \frac{\partial \mathbb{L}_{G}}{\partial f}$.

The dynamical equation and the Bianchi identities are given, respectively, by $d * \mathbb{F}=0$ and $d f=0$. Explicitly we have

$$
\begin{aligned}
\partial_{\theta}\left(f_{31} r \sin \theta\right)+\partial_{r}\left(f_{23} r^{2} \sin \theta\right) & =0 \\
\partial_{r}\left(f_{20} r\right)-\partial_{\theta} f_{10} & =0 \\
\partial_{\theta}\left(\mathbb{F}_{20} r \sin \theta\right)+\partial_{r}\left(\mathbb{F}_{10} r^{2} \sin \theta\right) & =0 \\
\partial_{r}\left(\mathbb{F}_{31} r\right)-\partial_{\theta} \mathbb{F}_{23} & =0
\end{aligned}
$$

The $G_{\mu \nu}$ tensor can be easily performed taking (in Cartesian coordinates) the following form

$$
G_{\mu \nu}=\left(\begin{array}{cccc}
-1+f_{10}^{2}+f_{20}^{2} & 0 & 0 & f_{20} f_{23}+f_{13} f_{10} \\
0 & 1+f_{31}^{2}-f_{10}^{2} & f_{32} f_{31}-f_{20} f_{10} & 0 \\
0 & f_{32} f_{31}-f_{20} f_{10} & 1+f_{32}^{2}-f_{20}^{2} & 0 \\
f_{20} f_{23}+f_{13} f_{10} & 0 & 0 & 1+f_{13}^{2}+f_{23}^{2}
\end{array}\right) .
$$


Notice that we will not take into account general powers of the geometrical Lagrangian (1), as for example $L_{g}=\left|\operatorname{det} G_{\mu \nu}\right|^{\frac{u}{2}}$, because by taking the 'square root form' $(u=1)$ the Lagrangian linearizes (as in the Yang-Mills case), and the solutions can be easily compared to other cases in the literature [1].

The physical magnetic field will be directly involved with the spin (physical electric fields lead null matrix element contribution to the cross section), consequently we must take $f_{13}=f_{23}=0$. The resulting Lagrangian is

$$
\sqrt{\left|\operatorname{det} G_{\mu \nu}\right|}=(2 \lambda)^{2}\left(1-f_{20}^{2}-f_{10}^{2}\right)
$$

and

$$
\mathbb{F}_{20}=-2(2 \lambda)^{2} f_{20}, \quad \mathbb{F}_{10}=-2(2 \lambda)^{2} f_{10}
$$

In this case the set of equations $(8,9)$ reduces to

$$
\begin{aligned}
\partial_{r}\left(f_{20} r\right)-\partial_{\theta} f_{10} & =0 \\
\partial_{\theta}\left(\mathbb{F}_{20} r \sin \theta\right)+\partial_{r}\left(\mathbb{F}_{10} r^{2} \sin \theta\right) & =0
\end{aligned}
$$

that leads immediately to the following solution for the physical magnetic fields

$$
f_{10}=-\frac{\varphi_{23}}{j}=\frac{2 \mu}{r^{3}} \cos \theta, \quad f_{20}=\frac{\varphi_{13}}{j}=\frac{\mu}{r^{3}} \sin \theta
$$

and

$$
\mathbb{F}_{10}=-\frac{\Phi_{23}}{j}=-2(2 \lambda)^{2} \frac{2 \mu}{r^{3}} \cos \theta, \quad \mathbb{F}_{20}=\frac{\Phi_{13}}{j}=-2(2 \lambda)^{2} \frac{\mu}{r^{3}} \sin \theta
$$

From the above equations the $h_{a}$ vector can be easily computed from the dynamical equation $[5,6,7]$ for the dual of the torsion field

$$
d h=-\lambda^{*} f
$$

We obtain

$$
\begin{aligned}
h_{3} \omega^{3} & =h_{\varphi} d \varphi \\
\text { with } \quad h_{\varphi} & =-\lambda \frac{\mu \sin ^{2} \theta}{r} \quad \text { or well } \rightarrow h_{3}=-\lambda \frac{\mu \sin \theta}{r^{2}}
\end{aligned}
$$




\section{CROSS-SECTION}

We consider the spin flip of a neutrino coming in the $z$-direction over a fixed source (e.g. a hadron). We now assume, as usual: $\psi_{i}=N_{i} u\left(k_{i}, \uparrow\right) e^{-i k_{i} x}$ and $\psi_{f}=N_{f} u\left(k_{f}, \downarrow\right) e^{-i k_{f} x}$ then, the transition amplitude is

$$
S_{f i}=\delta_{f i}-\frac{i}{4}\left(\frac{j \lambda}{d}\right) \int d^{4} x \bar{\psi}_{f} \gamma_{5} \gamma^{\mu} h_{\mu} \psi_{i}
$$

The invariant amplitude is given by the matrix element

$$
\mathcal{M}=\frac{i}{4} j_{5}^{\mu} h_{\mu}(q)
$$

where the axial current

$$
j_{5}^{\mu}=\bar{u}_{f} \gamma_{5} \gamma^{\mu} u_{i}
$$

in our case takes the form

$$
\bar{u}_{f}(p \uparrow) \gamma_{5} \gamma^{3} u_{i}(p \downarrow)
$$

We also have

$$
h_{\mu}(q)=\int e^{i q \cdot x} h_{\mu}(x) d^{3} x
$$

Considering $h_{3}(x)=-\lambda \frac{\mu \sin \theta}{r^{2}}$ and therefore using

$$
\gamma^{5} \gamma^{3} h_{3}(x)=\gamma^{5}\left(\gamma^{x} \sin \varphi+\gamma^{y} \cos \varphi\right)(d-1) \frac{\mu}{r^{2}}
$$

where the geometrical relations $d \varphi=\frac{1}{x^{2}+y^{2}}(x d y-y d x)$ and $r \sin ^{2} \theta d \varphi=(\cos \varphi d y-\sin \varphi d x)$ were introduced in order to change the tetrad basis to the coordinate one, the axial current in this case can be written exactly as

$$
\bar{u}_{f}(p, \uparrow) \gamma_{5} \gamma^{3} h_{3}(x) u_{i}(p, \downarrow)=\frac{p_{z}}{(E+m)}(d-1) \frac{\mu}{r^{2}}
$$

(notice that the Fourier transform was not performed yet). Then the matrix element is immediately written as

$$
\mathcal{M}=2 \frac{(d-1) \mu}{\pi^{2}(E+m)} \ln \left|\frac{q}{q_{\min }}\right|,
$$

where $m$ is the neutrino mass. In the low momentum transfer or elastic scattering limits (as in the computation of Ref. $[2,16])$ we obtain

$$
\mathcal{M} \simeq \frac{(d-1) \mu}{\pi^{2}(E+m)} \ln \left|\frac{2\left(E^{2}-m^{2}\right)(1-\cos \beta)}{q_{\min }^{2}}\right|
$$


with $\beta$ the scattering angle and the $q_{\min }$ can be associated to a suitable cutoff $\Lambda$ (see below).

From [8] we know that the Dirac equation derived from the same geometry of the spacetime in our unified model reads

$$
\left[i \gamma^{\alpha}\left(\nabla_{\alpha}+j \frac{1-d}{d} \gamma_{5} h_{\alpha}\right)-\frac{m c}{\hbar}\right] \psi=0
$$

where $j$ is a parameter of a geometrical origin (as we pointed out before) and $d$ is the spacetime dimension. This formula (28) is, as expected, completely analogous to other similar expressions on the literature coming from non standard theories involving torsion with minimal coupling.

The expression for the differential cross section can be easily shown to be

$$
\frac{d \sigma}{d \Omega}=\left(j \frac{1-d}{d}\right)^{2} \frac{E^{2}}{(2 \pi \hbar c)^{2}}|\mathcal{M}|^{2}
$$

From this we obtain the important result

$$
\sigma_{\nu}^{f l i p}=\left(\frac{j \mu m c}{4 \hbar}\right)^{2}\left(\frac{(1-d)^{2}}{\pi^{2} d}\right)^{2} \frac{E^{2}}{\left(E+m c^{2}\right)^{2}}\left[\ln \left|\frac{q^{2}}{q_{\min }^{2}}\right|\right]^{2}
$$

Notice the important fact that a similar expression was obtained in [2] for the anomalous angular momentum (with anomalous momentum, in the notation of $[2], \kappa \sim\left(j \frac{1-d}{d}\right)(d-1) \mu$ ).

In the elastic limit or the low momentum transfer we obtain

$$
\sigma_{\nu}^{f l i p} \approx\left(\frac{j \mu m c}{4 \hbar}\right)^{2}\left(\frac{(1-d)^{2}}{\pi^{2} d}\right)^{2} \frac{E^{2}}{\left(E+m c^{2}\right)^{2}}\left[\ln \left|\frac{2\left(E^{2}-m^{2} c^{4}\right)(1-\cos \beta)}{q_{\min }^{2}}\right|^{2}\right.
$$

Notice the explicit dependence on the energy, in sharp contrast with the string theoretical result [18] and the standard model one [16].

\section{SOLAR NEUTRINO SITUATION AND SPIN-FLIP}

To compare our results with the ones coming from string or the standard model theoretical considerations is useful to take the same notation and similar approximations that ref.[16,18] for example. Then, in the case of elastic scattering or low energy transfer, the probability of spin-flipping is given by

$$
\sigma_{\nu}^{f l i p}=A \frac{E^{2}}{\left(E+m c^{2}\right)^{2}}[\ln |B(1-\cos \beta)|]^{2}
$$


where we have defined

$$
A \equiv\left(\frac{j \mu m c}{4 \hbar}\right)^{2}\left(\frac{(1-d)^{2}}{\pi^{2} d}\right)^{2}, B \equiv \frac{2\left(E^{2}-m^{2} c^{4}\right)}{q_{\min }^{2}}
$$

Notice that in strong coincidence with the results of several experimental data $[12,19,17,1]$, the cross section (32) is explicitly energy dependent, and in our case we have, in addition, the geometrical parameter $j$ and the dimension $d$. In order to evaluate phenomenologically the problem, we must integrate $\sigma$ around the scattering angle $\beta$, as usual

$$
\begin{aligned}
& \sigma_{\nu}^{f l i p}(\beta)=A \frac{E^{2}}{\left(E+m c^{2}\right)^{2}} \overbrace{\int \sin \beta d \beta[\ln |B(1-\cos \beta)|]^{2}}^{\equiv I} \\
& \sigma_{\nu}^{f l i p}(\beta)=A \frac{E^{2}}{\left(E+m c^{2}\right)^{2}} 4\left[2+(\operatorname{Ln}(2))^{2}-\operatorname{Ln} 4+\log B(-2+\operatorname{Ln} 4+\log B)\right]
\end{aligned}
$$

where we have assigned the letter $I$ to the integral involving the scattering angle $\beta$, in order to compare in a clear manner with the corresponding string theoretical result or similar processes in the standard model. The explicit form of the above formula for $\sigma_{\nu}^{f l i p}(\beta)$ is

$$
\begin{aligned}
\sigma_{\nu}^{f l i p}(\beta) & =\left(\frac{j \mu m c}{4 \hbar}\right)^{2}\left(\frac{(1-d)^{2}}{\pi^{2} d}\right)^{2} \frac{E^{2}}{\left(E+m c^{2}\right)^{2}} . \\
& \cdot 4\left[1.09416+\operatorname{Ln}\left(\frac{2\left(E^{2}-m^{2} c^{4}\right)}{q_{\min }^{2}}\right)\left(\operatorname{Ln}\left(\frac{2\left(E^{2}-m^{2} c^{4}\right)}{q_{\min }^{2}}\right)-0.613706\right)\right]
\end{aligned}
$$

From the above results, it is important to note the following:

i) In formula (36), if we assume some astrophysical implications as in Ref.[16], the logarithmic terms can be bounded with values between 1 and 6 , depending on screening arguments, as is generally accepted. For the logarithmic terms close to $1, I$ is approximately 6. On the other hand, the string theoretical value of Ref. [18] takes the value $I=4.14$ (obtained numerically). This situation of taking the logarithmic energy dependent terms to be constant is at present questioned by the experimental point of view due to the arguments given in the Introduction.

ii) The $j$ parameter plays formally (at the cross section level) a role similar to that of the constant $\kappa$ of the string model with torsion of [18]. However in our approach, it is related to some physical "absolute field" (as $b$ in the Born-Infeld theory case, as we discuss in the Introduction) giving the maximum value that the physical fields can take into the spacetime (as the light velocity $c$ in the relativity theory). In such a case $j$ ("the absolute field") will be fixed to some experimental or phenomenological value. 
iii) The above results can be straighforwardly applied to the solar neutrino case (e.g. a neutrino emerging from the Sun). If we call $P(I)$ the probability that the neutrino suffers an helicity change of spin and $n$ being the number of scattering centers per unit of volume, the probability of a helicity flipping for the neutrino is

$$
P_{f l i p}=\int_{0}^{R_{\odot}} \sigma_{\nu}^{f l i p} n d l \approx \sigma_{\nu}^{f l i p} \bar{n} R_{\odot}
$$

where, to get the estimate, we have taken the $n$ value in the average $\bar{n}=10^{24} \mathrm{~cm}^{-1}$ and the Sun radius $R_{\odot}=10^{11} \mathrm{~cm}$. This expression has the geometrical parameter $j$ (see 36) as the link between the experimental data and the theory, as we will see soon.

\section{DISCUSSION AND EXAMPLES}

Now we will bring a few simple examples to discuss roughly the meaning of the results given in the previous sections in the light of some experimental results. At lower energies

of the neutrino $<0.1 \mathrm{MeV},[15,22,4]$ the magnetic momentum is around $10^{-11} \mu_{B}$ but at higher energies, but with the same bounded magnetic momentum of the neutrino, the cross section can be written as a function of the cross section for the spin flipping due to the $\mathrm{Z}^{0}$ exchange in the weak interaction

$$
\frac{d \sigma}{d \Omega}=\left(j \frac{1-d}{d}\right)^{2} \frac{2 G_{F}^{2} m^{2}}{3 \pi} \sin ^{4} \theta_{W}
$$

Notice that, if we consider the specific knowledge of the neutrino magnetic momentum, and the experimental probability (cross section) of the flipping then, the $j$ parameter (for instance $b$, the absolute field) will be partially fixed.

\section{A. Comparison with string-gravity model with torsion potential}

In [18], the cross section for the minimal coupling condition in the gravity string theory with torsion potential is

$$
\left.\sigma_{\nu}^{f l i p}(\beta)\right|_{\text {string }} \approx 8.28 \pi\left(\frac{9 G S m}{4 \hbar c^{2}}\right)^{2}
$$

where $G$ is the Newton constant and $S$ is the spin of the particle. 
In our theory, a rough comparison with the string model of [18] only can be made assuming, as we have pointed out before, the logarithmic terms in (36)close to 1 (or close to any other value between 1 and 6$)$, and $d=4$ :

$$
\left.\sigma_{\nu}^{f l i p}(\beta) \approx 1.5\left(j \frac{9}{\pi^{2} 4}\right)^{2} \sigma_{\nu}^{\text {flip }}(\beta)\right|_{\text {string }} .
$$

precisely in order to avoid the lack of energy dependence at high energies of the string theoretical result. Notice the existent relation between the $j$ parameter and the experimentally detected probability of flipping $P$. This fact will be pointed out in the Concluding Remarks.

\section{B. Cross sections energy-dependent and logarithmic terms}

As mentioned previously, the neutrino counts have been experimentally obtained for different energy ranges generating a clear preference on the cross sections with energy windows as is the case of the Bethe computations[2] and our own results computed here. This fact also evidently demands to review and to correct the claim about the constancy of the logarithmic terms involving the energy of the cross sections (as expression (36) or in the cross section computation of [2]).

\section{INTERACTION STRUCTURE, AXIONS AND NAMBU-GOLDSTONE BOSONS}

Theoretical arguments in a phenomenological context suggest that many symmetries of the nature involving fundamental particles are spontaneously broken. Having this fact into account, now we can show that a concrete relation between the axial vector $h_{\alpha}$ appearing of our model and the axion field $a$ exists: the reason of our claim is as follows. We focusing now in pseudoscalars: the first example are axions which from long time ago were proposed as a possible solution to the (strong) CP problem [20,21]. Actually, axions are only "pseudo Nambu-Goldstone bosons" in that the spontaneously broken chiral Peccei-Quinn symmetry $U_{P Q}(1)$ is also explicitly broken, providing these particles with a small mass

$$
m_{a}=0.60 e V \frac{10^{7} \mathrm{GeV}}{f_{a}}
$$


where, $f_{a}$ is an energy scale (sometimes called of Peccei-Quinn). This scale is related to the vev (vacuum expectation value) of the field which breaks explicitly the Peccei-Quinn symmetry $U_{P Q}(1)$.This scale is the main quantity to be astrophysically constrained due that the Nambu-Goldstone properties are directly related to it.. Notice that one can express limits on $f_{a}$ in terms of $m_{a}$. from the above equation due that it is specific to axions. To calculate the energy-loss rate of the axion fields from stellar plasmas, the interaction with the medium constituents must be specified. In general, the interaction with a fermion with mass $m_{f}$ is of 2 types, namely

$$
L_{1} \approx \frac{C_{f}}{2 f_{a}} \bar{\psi}_{f} \gamma^{\alpha} \gamma_{5} \partial_{\alpha} a \psi_{f}
$$

or

$$
L_{2} \approx-i \frac{m_{f} C_{f}}{f_{a}} \bar{\psi}_{f} \gamma_{5} a \psi_{f}
$$

where $\psi_{f}$ is the fermion and athe axion field, $C_{f}$ is a model-dependent coefficient of order unity and $g_{a f} \equiv \frac{m_{f} C_{f}}{2 f_{a}}$ plays the role of a Yukawa coupling. If we compare the interaction Lagrangians $L_{1}$ and $L_{2}$ and the interaction coming from the Dirac equation (28) derived in our unified model:

$$
L_{i n t} \approx \bar{\psi}_{f} \frac{1-d}{d} j \gamma^{\alpha} \gamma_{5} h_{\alpha} \psi_{f}
$$

we can easily see that precisely only the $L_{1}$ (derivative form) is related with $L_{\text {int }}$ provided that

$$
\partial_{\alpha} a \equiv h_{\alpha} \quad \text { and } \quad \frac{C_{f}}{2 f_{a}} \sim \frac{1-d}{d} j
$$

and $\mathrm{L}_{2}$ is automatically ruled out. This fact is largely consistent with the current research: as is well known, the interaction involving derivatives of the pseudoscalar is more fundamental in the sense that it respects the Nambu-Goldstone nature of these particles: e.g.: is invariant under $a \rightarrow a+a_{0}$. Contrarily, the pseudoscalar fashion of $L_{2}$ is supposed to be equivalent in the usual sense, because there are many technical problems and troubles when one try to calculate different processes where two Nambu-Goldstone bosons are attached to one fermion line ( for example an axion and a pion attached to a nucleon). [11]. Then, our remark here is that there exist a closed relation between the affine geometrical models with torsion as the analyzed here, where the lagrangian coming from the breaking of some symmetries (Goldstone) and the phenomenological observations concerning interaction with axions:. the frequently expected interaction term of derivative type naturally arises from our model. 


\section{NEUTRINO OSCILLATION AND ICECUBE DATA: CONSTRAINED THE TORSION EFFECT AT HIGHER ENERGIES}

As is well known, the phenomenon of oscillation gives a concrete and acceptable description of atmospheric neutrinos. Recently, the analysis of IceCube data [26] has provided the first significant detection $(>5 \sigma)$ of atmospheric neutrino oscillations at energies near the $25 \mathrm{GeV}$ oscillation maximum for vertical events. The measured oscillation parameters are in good agreement with results from other experiments that have measured the atmospheric oscillation parameters with high resolution at lower energies, some of them we have been mention in the Introduction. Then, the measurements agree with the theoretical predictions of the standard three-neutrino flavor oscillation framework that, in the context of the new physics effects (where torsion effects are included), means that the flipping effect has little significance at such energy window, as described by the IceCube data. However, (see [26] ) it is interesting to note that at lower energies, non oscillation effects seems to become a little bit more significant, given the possibility to constrain the torsion contribution to the neutrino problem and obtain concrete quantities in the cross section formula (36) for example. It is precisely, the scope of our actual research where the torsion effects must be compared and analyzed in the framework of the LHC also.

\section{CONCLUDING REMARKS}

In this letter the effect of the torsion field on the spin interaction was specifically analyzed in the case of a model corresponding to an unified field theory based on a generalized affine geometry, metric and with totally antisymmetric torsion field.. The main point to remark here is that, although the oscillation mechanism explain in a great meaning the solar neutrino problem, some little part of the trouble still remains and the discrepancy can be explained by the non standard interaction with the torsion field arising from this geometrical model. The main results of technical character are:

1) The cross section is energy dependent, even at high energies. It also depends on the spacetime dimension, and on a parameter $j$ of pure geometrical origin, probably associated to a scale or limiting value for the antisymmetric 2 -form field in the geometrical Lagrangian. 
2) This geometrical parameter plays a completely analogous role in our theory to that of the absolute $b$ field of the Einstein-Born-Infeld theory. The analogy appears because $j$ is associated to the 2 -form $f$ (the potential of the torsion field), homogenizing the units at the Lagrangian level and putting some limiting value to $f$, exactly as in the Born-Infeld case.

3) Contrarily to the string theory case of .[18] where there exist some $K$ that is a free parameter, the $j$ parameter is not completely free: there are physical constraints and phenomenological estimations coming from quantum and classical backgrounds that must indicate the specific freedom on his parameter (e.g. some bound over $j$ can be obtained from classical solutions plus Huges-Drever experiments)

4) The Lorentz symmetry and the minimal coupling are not violated due to the group structure of this specific model. The weak symmetry, as was explicitly shown here, is slightly violated.

5) Additionally, the equivalence principle, as was demonstrated in several references $[5,6,7,23,24,25]$, is not violated due to the totally antisymmetric character of the torsion field and the metric character of our theory.

6) Is clear that the cross-section computed here can not be directly compared with the cross section computed from the string gravity model with torsion potential of [18]. This fact is because the cross-section of .[18] has not energy dependent terms (even at high energies).

Will be very interesting to use the results from this new unified model in order to see the effects of the torsion in the treatment of the anomalies. This issue involving quantum field theoretical methods of non-local and not perturbative character (instantons, etc), are now under advanced research.

7) There exist a closed relation between the affine geometrical models with torsion and the phenomenological observations concerning interaction with axions:. the frequently expected interaction term of derivative type naturally arises from our model.

8) Because the new results from the IceCube data certainly ruled out at higher energies non-standard interactions, in the case of the atmospheric neutrinos the entire energy 
range must to be covered and analyzed to discern the effects of torsion from other non-standard interactions. These effects also must be constrained and compared with the possible torsion effects in the LHC framework.

Although the motivation of this work is clear we stress that the Standard Model of particle physics (SM) successful theory till today, evidently need to be revised due the observation of signs of a new physics beyond the scope of the SM. Which kind of a new physics can be expected from the astrophysical point of view? Evidently all aspects involving the inability of the SM to incorporate quantum gravity.

\section{ACKNOWLEDGEMENTS}

Many thanks are given to Professor Yu. P. Stepanovsky for my scientific formation and particularly to Dr. Victor I. Afonso for several discussions in the subject and his help in the preparation of this text. I am very grateful to Professor Xu Xin for introduce me into the affine geometrical models and gravitation and also to Professors J.W.F. Valle and F.J. Escrihuela for bring me very important references clearifying the subject of this paper and to Professor George Raffelt for very useful insights and discussions.

\section{REFERENCES}

[1] P. Anselmant \& al., 1993, GALLEX Colaboration, Phys. Lett B 314.

[2] H.Bethe, 1935, Proc. Cam. Phyl. Soc, 31, 108.

[3] S.Capozziello, G.Iovane, G.Lambiase \& C.Stornaiolo, 1999, Europhys.Lett. 46, 710715 .

[4] V. Castellani \& S. Degl'Innocenti, 1993, Astrophys. J. 402, 574.

[5] D.J. Cirilo-Lombardo, 2010, Int.J.Theor.Phys. 49 1288-1301.

[6] D.J. Cirilo-Lombardo, 2011a, Int.J.Theor.Phys. 50, 1699-1708.

[7] D.J. Cirilo-Lombardo, 2011b, Int.J.Theor.Phys. 50, 3621-3634.

[8] D.J Cirilo-Lombardo, Physics of Particles and Nuclei, 2013, Vol. 44, No. 5, pp. 848-865;

[9] D.J. Cirilo-Lombardo, 2007, J.Math.Phys. 48032301

[10] D.J. Cirilo-Lombardo, 2005, Class.Quant.Grav. 22 4987-5004. 
[11] Choi K., Kang K., \& Kim J.E., 1989, Phys. Rev. Lett. 62, 849

[12] R Davis, 1992, Proc. Int. Symp. on Neutrino Astrophysics (Takayama/Kamioka, 1992), ed . Y Suzuki and N. Nakamura (Tokio, Universal Academy) p.47.

[13] F.J. Escrihuela, O.G. Miranda, M.A. Tortola \& J.W.F. Valle, 2009, Phys.Rev. D80, 105009 ,

[14] F.J. Escrihuela, M. Tortola, J.W.F. Valle \& O.G. Miranda, 2011, Phys.Rev. D83, 093002

[15] M. Fukugita \& S. Yazaki, 1987,Phys. Rev. D 36, 3817.

[16] K. J. F. Gaemers \& al., 1989, Phys. Rev. D 40, 309-314.

[17] V. N. Gavrin, 1993, Proc. TAUP Workshop (19-21 Sept.1993, Gran Sasso Nat. Laboratory, Aquila, Italy).

[18] R. Hammond, 1996,Class.Quant.Grav. 13, 1691-1697.

[19] K. S. Hirata \& al., 1991 Phys. Rev Lett 66, 9.

[20] Peccei R.D.\& Quinn H.R., 1977a, Phys. Rev. Lett. 38, 1440

[21] Peccei R.D.\& Quinn H.R., 1977b,Phys. Rev. D 16, 1791;

[22] G. G. Raffelt, 1990, Astrophys. J. 365, 559.

[23] Yu Xin, 1996, "General Relativity on Spinor-Tensor Manifold", in: "Quantum Gravity - Int.School on Cosmology \& Gravitation", XIV Course. Eds. P.G. Bergman, V. de. Sabbata \& H.J. Treder, pp. 382-411, World Scientific.

[24] Yu Xin, 1989, Ap.SS 154, 321.

[25] Yu Xin, 1993 Ap.SS 202, 237.

[26] Measurement of Atmospheric Neutrino Oscillations with IceCube, IceCube Collaboration, arXiv:1305.3909 
[1] Geometrical Lagrangians with generalized powers are sometimes convenient when the symmetry of the considered coordinates coincides with the symmetry of the group structure of the spacetime manifold, as in that cases $\left|\operatorname{det} G_{\mu \nu}\right|$ becomes a perfect square and the Lagrangian is automatically linearized (becomes of 'Yang-Mills-type'), with the consequent destruction of the nonlinear and nonlocal character of the solutions [6] 